\title{
JUSTYNA WOROCH
}

Université Adam Mickiewicz, Poznań

\section{LES REFORMULATIONS RÉPARATRICES DANS L'INTERPRÉTATION DE CONFÉRENCE}

\begin{abstract}
A bstra ct. Woroch Justyna, Les reformulations réparatrices dans l'interprétation de conférence [Repair reformulations in conference interpreting]. Studia Romanica Posnaniensia, Adam Mickiewicz University Press, Poznań, vol. XXXVII/2: 2010, pp. 123-130. ISBN 978-83-232-2189-0. ISSN 0137-2475. DOI 10.2478/v10123-010-0020-4.

The aim of this paper is to deal with the notion of repair reformulations (fr. reformulations réparatrices) investigated mainly by the conversational analysis researchers but adapted here to the situation of conference interpreting. The author analyses a couple of examples of repairs which appear in the texte produced by the speaker as well as those relevant for the interpreter's delivery.
\end{abstract}

\section{INTRODUCTION}

La définition courante de reformulation, telle qu'on peut la retrouver par exemple dans Le Trésor de la Langue Française Informatisé, est la suivante : « nouvelle formulation qui reproduit autrement ce qui a été dit ». Il n'est pas difficile à imaginer beaucoup de contextes dans lesquels on peut avoir affaire aux reformulations. Il existe des procédés de reformulation propres à l'oral et à l'écrit. Il peut s'agir de reformuler sa propre parole ou la parole de l'autre. Ceci peut être un processus spontané ou au contraire, réfléchi et bien structuré. Finalement, pour donner à cette notion une dimension traductologique, la reformulation est envisageable non seulement au sein d'une langue, mais aussi bien dans le contact de deux langues.

Les quelques contextes précités (cette liste n'est en aucun cas exhaustive) avaient intéressé les chercheurs différents et les ont menés à élaborer des acceptions très diversifiées de ce terme.

Dans le cadre de la présente communication nous allons nous pencher sur le phénomène de reformulation dans l'interprétation de conférence. Étant donné le fait que cette situation de communication suppose la meilleure transmission du sens possible, il nous semble que les reformulations réparatrices, appelées également " (auto-)corrections » (Gülich et Kotschi, 1987) y seront retrouvables. Notre brève analyse va s'appuyer sur la comparaison des discours prononcés en français, enregistrés pendant la conférence internationale intitulée « Od Solidarności do Wolności », tenue à Varso- 
vie le 29 et 30 août 2005, et leurs interprétations simultanées en polonais, effectuées sur place par l'interprète professionnel. Les intervenants enregistrés sont Jean-Bernard Raimond (désormais JBR), ancien ministre des affaires étrangères en France et cardinal Jean-Marie Lustiger (désormais JML). Les discours durent environ une demi-heure chacun.

\section{CADRE THÉORIQUE}

Comme nous venons de signaler, la notion de reformulation attire l'attention des chercheurs aux orientations scientifiques différentes. Leurs travaux ont en commun le fait de se pencher sur des concepts dans lesquels il s'agit d'établir des relations d'équivalence sémantique ou formelle avec un ou plusieurs énoncés précédents (cf. Doggen, 2008). Nous pensons ici notamment aux travaux de Claire Martinot (Martinot, 2003 et 2008) qui place cette notion au cœur de la réflexion sur l'acquisition de la langue maternelle, aux analyses de Fuchs (1982 et 1994) qui construit une théorie énonciative de paraphrase, aux procédés de reformulation et leurs objectifs pragmatiques, se répercutant dans le discours didactique et dans l'interaction pédagogique (notons de passage la notion de glose, étudiée par Authier-Revuz et Steuckardt, cf. Doggen, 2008). Il ne faut pas oublier encore un autre procédé, celui d'adaptation. Et finalement l'opinion, se situant à un niveau beaucoup moins technique de réflexion, que toute traduction peut être vue comme reformulation (Fuchs, 1982).

Reste à placer la reformulation dans la perspective la plus proche de notre champ d'intérêt, celui de l'oral. Dans les travaux des chercheurs en interprétation, d'un côté elle est vue en tant que stratégie d'apprentissage de l'interprétation (cf. à titre d'exemple Gillies, 2001) et de l'autre côté, en tant que technique en interprétation de conférence (cf. par exemple Jones in Gillies, 2001, p. 82 qui dit : « Reformulation, in its various forms, is one of the most useful tools the simultaneous interpreter has »). Comme l'atteste le spécialiste polonais en interprétation, Kopczyński (1998, p. 82) « [...] ulotny charakter komunikatu, jakość instalacji, hałas, widoczność, sposób i tempo wygłaszania komunikatu, ograniczenie pamięci thumacza [...] Wszystko to może przyczynić się do opuszczania, dodawania, skracania, streszczania lub parafrazowania partii tekstu przez thumacza [...] Czy robienie tego jest błędem ? Są znawcy przedmiotu, którzy sądzą, że operacje te stanowią istotę tłumaczenia ustnego, odróżniająca go od thumaczenie pisemnego ».

En voulant observer si des procédés de reformulations réparatrices sont retrouvables dans les discours oraux destinés à être interprétés et dans les interprétations ellesmêmes, nous allons faire recours aux travaux pionniers des années 80 dans le domaine de l'analyse conversationnelle. Nous nous rendons compte que l'objet analysé dans ces travaux diffère du nôtre [l'interaction verbale de deux interlocuteurs dans le cas de Gülich et Kotschi (1987), de Gaulmyn (in : Gülich et Kotschi, 1987) ou bien la 
description du français parlé par le biais d'un corpus constitué des interventions des gens à qui on a imposé des sujets à traiter dans le cas de Coste, 1986], cependant ils élaborent les outils de description des phénomènes propres à l'oral qui nous semblent suffisament généraux pour pouvoir être adaptés à l'analyse d'un corpus ayant tout de même quelques traits en commun avec les leurs.

Les chercheurs allemands aboutissent à l'acception suivante du terme de reformulation :

Nous désignons ces actes [le rephrasage, la paraphrase et la correction] par le terme général d'"actes de reformulation" (ou simplement "reformulations"). Ils ont ceci en commun que leur réalisation présuppose la relation $\mathrm{xRy}$, de sorte que dans la structure du texte on trouve, comme résultat de la réalisation de chacun de ces trois actes, deux segments que nous appelons "énoncé-source" et "énoncé reformulateur". Le rephrasage, la paraphrase et la correction se distinguent a) par la nature de $\mathrm{R}$ et b) par des sous-ensembles de "marqueurs de reformulation" [...] (Gülich et Kotschi, 1987, pp. 30-31).

Dans le cadre des recherches menées par de Gaulmyn (in : Gülich et Kotschi, 1987) apparaissent les notions des auto-reformulations et des hétéro-reformulations (en fonction du nombre de sujets qui participent à leur création), elle peuvent être immédiates ou retardées. Coste se concentre sur les auto-interruptions et reprises.

\section{ANALYSE}

Nous divisons les exemples qui suivent en deux grandes catégories. Dans un premier temps, nous observons les reformulations réparatrices au sein des discours prononcés par les intervenants. Il est intéressant de voir si ces reformulations, une fois détectées dans les discours originaux, sont interprétées et de quelle manière. Deuxièmement nous passons à celles retrouvables dans les discours d'interprète. Après avoir décrit leur structure, nous allons essayer de comprendre leur fonction.

\section{a. Reformulations au sein du discours original et leurs interprétations}

Selon Gülich et Kotschi (1987, p. 42), « Dans les cas typiques de correction la justesse de l'énoncé-source se trouve entièrement ou partiellement "annulée" par la valeur de l'énoncé reformulateur ». Outre la relation sémantique existant entre énoncé-source et énoncé reformulateur, les chercheurs concentrent leur attention sur le type de marqueur qui est utilisé pour indiquer cette relation. Ils précisent que « Le marqueur est une trace, laissée dans le discours par le travail conversationnel du locuteur » (Gülich et Kotschi, 1987, p. 44) tout en étant conscients que ces marqueurs ne doivent pas forcément être verbaux, mais aussi prosodiques.

Dans notre premier exemple (1), qui provient du discours original et constitue un lapsus, nous pouvons voir des phenomènes suivants : l'orateur débute sa séquence avec l'unité la Pologne militaire pour changer tout de suite le qualificatif en millénaire. L'annulation sémantique est totale, entre les deux unités il n'y a pas de marqueur 
de reformulation explicite. On pourrait donc croire que la correction est déjà achevée, cependant vient tout de suite après la formule excusez-moi suivie de la reprise de tout l'énoncé reformulateur corrigé, c'est-à-dire la Pologne millénaire. À notre sens cette formule de politesse peut être traitée ici en tant que marqueur de reformulation.

(1)

\begin{tabular}{|l|c|}
\hline \multicolumn{1}{|c|}{ Intervenant : JBR } & $\begin{array}{c}\text { Interprète : professionnel en situation } \\
\text { réelle }\end{array}$ \\
\hline $\begin{array}{l}\text { la Pologne militaire, millénaire, } \\
\text { excusez-moi, la Pologne millénaire } \\
\text { était soudainement libre }\end{array}$ & $\begin{array}{l}\text { 194. Tysiącletnia Polska była } \\
\text { suwerenna i wolna }\end{array}$ \\
\hline
\end{tabular}

Cette auto-reformulation, qui a une fonction d'auto-correction (cf. Schegloff, Jefferson et Sacks in: Gülich et Kotschi, 1987) n'a pas laissé de traces dans le discours d'interprète qui ne donne aux participants polonais de la conférence que la « version finale » de l'orateur, à savoir : tysiącletnia Polska.

Dans le cas suivant (2) l'intervenant commence sa séquence avec l'unité le travail, pour la changer tout de suite après en les travailleurs. Entre l'énoncé-source et l'énoncé reformulateur il n'y a pas de marqueur verbal de reformulation. Du point de vue du sémantisme, l'annulation n'est pas totale. À la lumière des remarques de Coste (1986, p. 131) il s'agirait plutôt d'une auto-interruption avec reprise-modification («le locuteur interrompt le déroulement syntaxique de son énoncé et reprend en le(s) modifiant un ou des éléments précédant immédiatement la rupture. [...] il n’y a pas de remise en cause de la trajectoire syntaxique majeure, même si peuvent s'opérer par ce biais des corrections de trajectoire $»)$.

(2)

\begin{tabular}{|l|c|}
\hline \multicolumn{1}{|c|}{ Intervenant : JML } & $\begin{array}{c}\text { Interprète : } \\
\text { professionnel en situation réelle }\end{array}$ \\
\hline $\begin{array}{l}\text { Et le travail... les travailleurs, } \\
\text { en revendiquant leur dignité }\end{array}$ & $\begin{array}{c}\text { 120. To ludzie pracy żądając uznania } \\
\text { i godności }\end{array}$ \\
\hline
\end{tabular}

L'interprétation, comme dans le cas de l'exemple numéro 1 , ne reflète pas cette opération.

L'exemple suivant (3) constitue à nos yeux un autre cas de figure d'auto-interruption, qui devient, dans le fond, une auto-correction. Selon la typologie plus détaillée de Gülich et Kotschi, il s'agirait, nous semble-t-il, d'une paraphrase-exemplification, où « il s'agit de restreindre l'extension du référent de l'énoncé-source » (Gülich et 
Kotschi, 1987, p. 41). Du point de vue de sa structure, il n'y a pas de marqueur verbal de reformulation.

(3)

\begin{tabular}{|l|c|}
\hline \multicolumn{1}{|c|}{ Intervenant : JBR } & \multicolumn{1}{|c|}{$\begin{array}{c}\text { Interprète : professionnel en situation } \\
\text { réelle }\end{array}$} \\
\hline $\begin{array}{l}\text { l'URSS n'interviendrait plus aux } \\
\text { affaires intérieures des pays d'Orient... } \\
\text { d'Europe Orientale et Centrale }\end{array}$ & $\begin{array}{c}\text { 314. ZSRR nie będzie interweniować } \\
\text { w wewnętrzne sprawy } \\
\text { 315. krajów Europy Środkowej } \\
\text { i Wschodniej }\end{array}$ \\
\hline
\end{tabular}

On peut considérer que de nouveau l'interprète transcode correctement le travail auto-correctif de l'intervenant car il n'interprète que l'énoncé reformulateur de cette séquence (3) (notons seulement de passage le changement d'ordre de cette énumération, Orientale et Centrale contre Środkowa $i$ Wschodnia).

La dernière auto-interruption avec reprise-modification (4) analysée dans le cadre de cette présentation ne comporte pas non plus de marqueur verbal de reformulation. Cependant nous pouvons voir qu'il s'agit du travail rectificateur de l'intervenant, portant, nous semble-t-il, sur une certaine maladresse de style qui, grâce à cette coupure, est évitée.

\begin{tabular}{|c|c|}
\hline Intervenant : JBR & $\begin{array}{c}\text { Interprète : professionnel en situation } \\
\text { réelle }\end{array}$ \\
\hline $\begin{array}{l}\text { Monsieur le Président, Monsieur le } \\
\text { Président, Messieurs les Ministres, } \\
\text { Monsieur le Ministre Geremek, } \\
\text { Monsieur le Cardinal, et si je me } \\
\text { permets... si je peux me permettre, } \\
\text { je dirai chers amis, pour des raisons } \\
\text { diverses, liées à la Pologne }\end{array}$ & $\begin{array}{l}\text { 1. Panie Prezydencie, Panie } \\
\text { Prezydencie, } \\
\text { 2. Panowie Ministrowie, } \\
\text { 3. Panie Ministrze Geremek, } \\
\text { 4. Eminencjo, } \\
\text { 5. i, jeżeli Państwo pozwola, } \\
\text { chciałbym powiedzieć drodzy } \\
\text { przyjaciele, } \\
\text { 6. i mówię to z wielu powodów, } \\
\text { związanych zresztą z Polską }\end{array}$ \\
\hline
\end{tabular}

Pour ce qui est de l'interprétation de cette séquence, elle ne reprend pas ce travail correctif de l'orateur. L'interprète ne choisit qu'une formule pour rendre l'intention du locuteur. Cependant il renverse la perspective (moi / vous). 
b. Reformulations au sein du discours d'interprète

Après avoir vu (quelques-unes) des actions auto-correctives présentes dans le discours de l'orateur et retenues par l'interprète uniquement sous forme d'énoncés reformulateurs, essayons d'observer si l'interprète lui-même, à son tour, ajuste ses propos dans certains endroits, pour rendre plus clairement et fidèlement les propos français aux participants polonais.

Nous pouvons constater la présence de séquence du type énoncé-source / marqueur verbal de reformulation / énoncé reformulateur dans l'exemple qui suit (5) :

(5)

\begin{tabular}{|l|c|}
\hline \multicolumn{1}{|c|}{ Intervenant : JML } & \multicolumn{1}{c|}{$\begin{array}{c}\text { Interprète : } \\
\text { professionnel en situation réelle }\end{array}$} \\
\hline $\begin{array}{l}\text { le même danger existe de méconnaître } \\
\text { le réel de la condition humaine et de } \\
\text { sa dignité au benéfice des nouvelles } \\
\text { idéologies régnantes }\end{array}$ & $\begin{array}{c}\text { 230. zachodzi praktycznie to } \\
\text { samo ryzyko nieznajomości } \\
\text { rzeczywistości kondycji człowieka, } \\
\text { 231. i jego godności, } \\
\text { 232. na rzecz nowych, } \boldsymbol{c z y} \text { też nowo } \\
\text { panujących ideologii }\end{array}$ \\
\hline
\end{tabular}

Grâce à ce procédé, l'interprète a mieux rendu le sens de l'original, en réprécisant qu'il ne s'agit pas seulement des nouvelles idéologies quelconques, mais des nouvelles idéologies régnantes. Il a évité de commetre une faute de traduction, un faux-sens.

Dans les trois exemples qui suivent $(6,7,8)$, nous avons également affaire à des auto-corections de la part de l'interprète, toutes les trois sans marqueur verbal de reformulation. Dans l'exemple numéro 6 nous voyons la recherche du qualificatif plus approprié par rapport à l'original.

(6)

\begin{tabular}{|l|c|}
\hline \multicolumn{1}{|c|}{ Intervenant : JBR } & \multicolumn{1}{c|}{$\begin{array}{c}\text { Interprète : } \\
\text { professionnel en situation réelle }\end{array}$} \\
\hline $\begin{array}{l}\text { L'Europe Orientale et Centrale } \\
\text { comprennent immédiatement la portée } \\
\text { révolutionnaire de l'événement, les } \\
\text { Allemands les premiers }\end{array}$ & $\begin{array}{l}\text { 15. Mieszkańcy Europy Środkowej } \\
\text { i Wschodniej od razu zrozumiały, } \\
\text { 16. jak wielkie znaczenie, rewolucyjne, } \\
\text { ma to wydarzenie, } \\
\text { 17. Niemcy na czele to zrozumieli }\end{array}$ \\
\hline
\end{tabular}


Dans l'exemple suivant (7) deux phénomènes sont à observer. D'abord, une auto-correction portant sur le chiffre (la date), sous forme de cassure sur un mot plein (selon la typologie de Coste, cf. Coste 1986, p. 132), qui permet d'éviter un faux-sens. Ensuite, une modification à fonction nuançante (toujours au dire de Coste 1986) où l'interprète réussit à ajouter le qualificatif pierwsza à l'énoncé jego podróż do Polski.

(7)

\begin{tabular}{|l|c|}
\hline \multicolumn{1}{|c|}{ Intervenant : JML } & $\begin{array}{c}\text { Interprète : } \\
\text { professionnel en situation réelle }\end{array}$ \\
\hline $\begin{array}{l}\text { l'élection du pape Jean-Paul II en 1978 } \\
\text { et son premier voyage en Pologne }\end{array}$ & $\begin{array}{c}\text { 17. wybór papieża Jana Pawła II } \\
\text { w siedemdziesiátym dru... ósmym } \\
\text { roku, jego podróż do Polski... }\end{array}$ \\
18. pierwsza podróż do Polski
\end{tabular}

Finalement, nous pouvons analyser le dernier exemple (8) de cette courte série d'auto-corrections de l'interprète. Cette fois-ci nous notons qu'elle reçoit la forme d'une double répétition d'une unité (sit) qui témoignerait de la recherche pénible de formulation exacte en polonais de l'expression française rapport de force.

(8)

\begin{tabular}{|l|c|}
\hline \multicolumn{1}{|c|}{ Intervenant : JML } & \multicolumn{1}{c|}{$\begin{array}{c}\text { Interprète : } \\
\text { professionnel en situation réelle }\end{array}$} \\
\hline $\begin{array}{l}\text { Dans l'opinion mondiale aussi l'aspect } \\
\text { polémique commence aujourd'hui à être } \\
\text { largement répandu, mais en terme de } \\
\text { révolte, et de rapport de force }\end{array}$ & $\begin{array}{c}\text { 243. Ale również w skali światowej, } \\
\text { poli... polemika zaczyna się właśnie } \\
\text { upowszechniać } \\
\text { 244. ale upowszechniać jako bunt i sił... } \\
\text { sił... próba sit. }\end{array}$ \\
\hline
\end{tabular}

Notons de passage la présence d'un autre type de correction, qu'on aurait appelé provisoirement « auto-correction phonétique », où a lieu un faux-départ, une autointerruption pour éviter une mauvaise prononciation d'une unité donnée (ici : poli... polemika). 


\section{CONCLUSION}

Nous pouvons confirmer la présence dans notre corpus, provenant de la situation de communication particulière, celle de l'interprétation pendant une conférence internationale, des reformulations réparatrices. Elles se situent à deux niveaux différents. D'un côté il s'agit des reformulations dans le discours original, de l'autre côté nous pouvons constater la présence des auto-corrections dans le discours d'interprète.

La nature des auto-corrections dans le cadre du discours original paraît assez homogène, il s'agit de la recherche d'une meilleure formulation (exemples 1, 2, 4) du point de vue linguisitique ou d'une formulation plus précise (exemple 3) du point de vue informationnel. Leur interprétation ne retient que la version finale, autrement dit que l'énoncé reformulateur.

Les auto-corrections faites par l'interprète lui-même témoignent un contrôle de sens et de forme de son discours.

Nous nous rendons compte que pour pouvoir confirmer nos conclusions qui ne sont pour l'instant que provisoires, vu le nombre d'exemples analysés, il faut envisager les expérimentations ultérieures avec la participation d'un nombre plus important d'interprètes. Une analyse beaucoup plus approfondie des discours originaux est également prévue (la description plus détaillée du caractère de ces textes, la recherche des reformulations non seulement réparatrices mais ayant d'autres fonctions...) ainsi que des discours d'interprètes.

\section{BIBLIOGRAPHIE}

Coste, D. (1986). Auto-interruptions et reprises. DRLAV Revue de linguistique, Paroles inachevées, 34-35, 127-139.

Doggen, J. (2008). Reformulations didactiques : effets sur la prise de notes d'étudiants francophones. Dans M. Schuwer, M.-C. Le Bot, \& É. Richard, Pragmatique de la reformulation. Types de discours. Interactions didactiques. Rennes : Presses Universitaires de Rennes.

Fuchs, C. (1982). La paraphrase. Paris : Presses Universitaires de France.

Gillies, A. (2001). Ttumaczenie ustne. Poradnik dla studentów. Kraków : Krakowskie Towarzystwo Popularyzowania Wiedzy o Komunikacji Językowej « Tertium ».

Gülich, E. \& Kotschi, T. (1987). Les actes de reformulation dans la consultation : « La dame de Caluire ». Dans P. Bange (Ed.), L'analyse des interactions verbales. La dame de Caluire: une consultation. Actes du colloque tenu à l'Université de Lyon 2 du 13 au 15 décembre 1985, Berne : Peter Lang.

Kopczyński, A. (1998). Tłumacz konferencyjny - cień czy intruz ? Dans J. Hartzell, A. Czołnowska, \& Z. Pawlak, Ocena tlumaczenia ustnego. Materiały Konferencji Naukowej OBiSP. Łódź : Ośrodek Badań i Studiów Przekładowych Uniwersytetu Łódzkiego.

Le Trésor de la Langue Française Informatisé. (s.d.). Récupéré sur http://www.atilf.fr

Martinot, C. (2003). La reformulation : un principe universel d'acquisition. Paris : Éditions Kimé.

Martinot, C., Gerolimich, S., Paprocka-Piotrowska, U., \& Sowa, M. (2008). Reformuler pour acquérir sa langue maternelle ? Investigation auprès d'enfants français, italiens et polonais de 6,8 et 10 ans. In M. Schuwer, \& M.-C. Le Bot, Pragmatique de la reformulation. Types de discours. Interactions didactiques. Rennes : Presses Universitaires de Rennes. 